\title{
Incomplete Data Problems in X-Ray Computerized Tomography
}

\section{Truncated Projections and Region-of-Interest Tomography}

\author{
Alfred K. Louis * and Andreas Rieder * \\ Fachbereich Mathematik, MA 6-1, Technische Universität Berlin, \\ Strasse des 17. Iuni 136, D-1000 Berlin 12
}

\begin{abstract}
Summary. In the present paper we study truncated projections for the fanbeam geometry in computerized tomography. First we derive consistency conditions for the divergent beam transform. Then we study a singular value decomposition for the case where only the interior rays in the fan are provided, as for example in region-of-interest tomography. We show that the high angular frequency components of the searched-for densities are well determined and we present reconstructions from real data where the missing information is approximated based on the singular value decomposition.
\end{abstract}

Subject Classifications: AMS(MOS): 65R10; CR: 61.2.

\section{Introduction}

The mathematical model describing $\mathrm{x}$-ray computerized tomography is the socalled $\mathrm{x}$-ray transform. In two dimensions it coincides with the Radon transform which assigns a real-valued density distribution $f$ its integrals over all straight lines. For an overview of the state-of-art results see Natterer [9].

Describing the unit vectors $\omega \in S^{1}$ by $\omega=\omega(\varphi)=(\cos \varphi, \sin \varphi)^{\top}$ we get with $\omega^{\perp}=\omega\left(\varphi+\frac{\pi}{2}\right)=(-\sin \varphi, \cos \varphi)^{\top}$ the standard parametrization of the rays leading to the Radon transform

$$
\mathscr{R} f(s, \omega)=\int_{\mathbf{R}} f\left(s \omega+t \omega^{\perp}\right) d t
$$

with $s \in \mathbf{R}$. This notation is best suited for the parallel geometry where $\mathrm{x}$-ray source and detector are moved to sample all parallel lines over the patient.

\footnotetext{
* The work of the authors was supported by the Deutsche Forschungsgemeinschaft under grant Lo 310/2-4 EVA-STAR (Elektronisches Volltextarchiv - Scientific Articles Repository) 
This was used in the first scanners and has the disadvantage that the sampling time is far to long for the resolution which is now achievable. Therefore in the so-called fan-beam geometry a single $\mathrm{x}$-ray tube is mounted on a gantry and moved around the patient. Opposite that tube is a set of detectors sampling at an instance all the rays emanating from the tube. In that geometry we use the divergent beam transform parametrized by two directions or simply by two angles $\alpha$ and $\beta$. The source position is $d \omega(\alpha+\pi / 2)$ where $d$ denotes the radius of the source circle. If we scale our object such that it is compactly supported in the unit circle then $d$ is larger than 1, in practical situations between 2.5 and 3.5. Furthermore $\beta$ denotes the angle of a ray with the center ray connecting the source position with the origin 0 . Between the two parametrizations there is the relation

$$
\mathscr{P} f(\alpha, \beta)=\mathscr{R} f(d \sin \beta, \omega(\alpha+\beta)),
$$

with $\alpha \in[0,2 \pi[$ and $\beta \in[-\Phi, \Phi]$, where $\Phi=\arcsin (1 / d)$. In [2] the operator $\mathscr{P}$ is called the divergent beam transform. Based on this coordinate transform fast reconstruction algorithms are developped which allow for parallel processing and hence real-time inversion, as long as the data are sampled on a regular mesh.

In [6] we performed a stability analysis based on a singular value decomposition of the limited angle transform when restrictions are posed on the angular range $\omega=\omega(\varphi)$. In the present paper we study the region-of-interest problem when only the subset of diagnostic relevance is covered by $\mathrm{x}$ rays. When for example the spinal chord is examined there is no point in wasting radiation on the rest of the abdomen. In our notation (1.2) this means that we study restrictions on $\beta$ and hence on $s$ in the original parametrization. First one has to mention a nonuniqueness result by K.T. Smith [2] saying that even in the region of interest it is not possible to recover the density uniquely and that the functions in the nullspace are almost constant there, thus allowing for detecting the boundaries of the organs but not their actual densities. In the present paper we first derive consistency conditions for the fan-beam geometry in form of a singular value decomposition of the divergent-beam transform. Then we repeat the nonuniqueness result for the truncated projections and we reduce the singular value decomposition for this interior transform to the eigenvalue problem of some compact operators. The stability problem is then discussed based on these operators. Finally we present reconstructions from real data. They show that the sophisticated regularization of this ill-posed problem gives extremly good results.

\section{Consistency Conditions for the Fan-Beam Geometry}

We consider the Radon transform as mapping between $L_{2}(\Omega)$ and $L_{2}\left(Z, w^{-1}\right)$ where $\Omega$ is the unit disk, $Z=[-1,1] \times S^{1}$ is the unit cylinder and $w$ is the weight $w(s)=\left(1-s^{2}\right)^{1 / 2}$. 
Lemma 2.1. Let $\mathscr{R}: L_{2}(\Omega) \rightarrow L_{2}\left(Z, w^{-1}\right)$. Then $\mathscr{R}$ has the singular value decomposition

with

$$
\left\{v_{m, l}, u_{m, l} ; \sigma_{m, l}\right\}
$$

where

$$
m \geqq 0, \quad-m \leqq l \leqq m \text { and } \quad m+l \text { even }
$$

$$
\begin{gathered}
v_{m, l}(x)=\pi^{-1 / 2}(m+1)^{1 / 2}|x|^{l} P_{(m-l) / 2}^{(0, l)}\left(2|x|^{2}-1\right) e^{i l \arg (x)}, \\
u_{m, l}(s, \omega)=\pi^{-1} w(s) U_{m}(s) e^{2 l \varphi}, \\
\sigma_{m, l}=2 \pi^{1 / 2}(m+1)^{-1 / 2},
\end{gathered}
$$

where the $P_{n}^{(\alpha, \beta)}$ are the Jacobi-polynomials and the $U_{m}$ are the Chebyshev-polynomials of the second kind.

Proof. See [5, 7, 9].

The singular functions $\left\{v_{m, l}\right\}$ form a complete orthonormal system in the closure of the range of the adjoint operator which is equal to the orthogonal complement of the null space, and the $\left\{u_{m, l}\right\}$ are a complete orthonormal system in the closure of the range of the operator. They have the following property

$$
\mathscr{R} v_{m, l}=\sigma_{m, l} u_{m, l} \quad \text { and } \quad \mathscr{R}^{*} u_{m, l}=\sigma_{m, l} v_{m, l} .
$$

With this result we can characterize the range of the transform.

Corollary 2.2. Let $\mathscr{R}: L_{2}(\Omega) \rightarrow L_{2}\left(Z, w^{-1}\right)$. Then $g \in L_{2}\left(Z, w^{-1}\right)$ is in the closure of the range of $\mathscr{R}$ if and only if

$$
g(s, \omega)=w(s) \sum_{m=0}^{\infty} U_{m}(s) \sum_{\substack{l=-m \\ m+l \text { even }}}^{m} g_{m, l} e^{i l \varphi} .
$$

The function $g$ is in the range of $\mathscr{R}$ if it has the representation (2.4) and

$$
\sum_{m=0}^{\infty}(m+1) \sum_{\substack{l=-m \\ m+l \text { even }}}^{m}\left|g_{m, l}\right|^{2}<\infty .
$$

Proof. From Lemma 2.1 follows the representation of the functions in the closure of the range of $\mathscr{R}$. Applying Picard's criterion yields (2.5).

As further corollary we find Helgason's consistency conditions characterizing the closure of the range of $\mathscr{R}$ by the three conditions

$$
\begin{aligned}
& g(s, \omega)=0 \quad \text { for }|s|>1, \\
& g(s, \omega)=g(-s,-\omega),
\end{aligned}
$$

$\int g(s, \omega) s^{k} d s$ is a homogeneous polynomial of degree $k$ in $\omega$. 
Regularity assumptions on $g$ dependent on $f$ in suitable Sobolev spaces correspond to (2.5).

In the next step we want to restate this result for the fan-beam geometry. We introduce the notation

$$
\begin{gathered}
W(\beta)=d \cos \beta w^{-1}(d \sin \beta), \\
V_{m}(\beta)=\pi^{-1} w(d \sin \beta) U_{m}(d \sin \beta),
\end{gathered}
$$

and

$$
Q=[0,2 \pi] \times[-\Phi, \Phi] .
$$

We derive the following consistency conditions for the fan beam geometry, see also [4]. With $\alpha$ we denote the angle between the $x_{2}$-axis and the line connecting the origin with the $\mathrm{x}$-ray source.

Theorem 2.3. Let $\mathscr{P}: L_{2}(\Omega) \rightarrow L_{2}(Q, W)$. Then $g$ is in the closure of the range of $\mathscr{P}$ if and only if

$$
g(\alpha, \beta)=\sum_{l \in \mathbf{Z}} e^{i l(\alpha+\beta)} \sum_{k=0}^{\infty} g_{l, k} V_{|l|+2 k}(\beta)
$$

and $\mathrm{g}$ is in the range of $\mathscr{P}$ if it has the representation (2.6) and

$$
\sum_{l \in \mathbf{Z}} \sum_{k=0}^{\infty}(|l|+2 k)\left|g_{l, k}\right|^{2}<\infty .
$$

Proof. This is a consequence of Lemma 2.1 and a reorganization of the double sum.

In contrast to the parallel geometry there are no such simple analogues as Helgason's consistency conditions. We have for example

$$
\int_{0}^{2 \pi} g(\alpha, \beta) d \alpha=2 \pi \sum_{k=0}^{\infty} g_{0, k} V_{2 k}(\beta) .
$$

\section{Indeterminacy and Singular Value Decomposition of the Interior Problem}

In the sequel we consider truncated projections in the following sense. We assume that $\mathscr{P} f(\alpha, \beta)$ is given for all $\alpha \in[0,2 \pi]$ and

$$
|\beta| \leqq B<\Phi .
$$

We denote by

$$
Q_{B}=[0,2 \pi] \times[-B, B]
$$

the set where the data are given. We first have to state the following result. 
Lemma 3.1. Let $\mathscr{P} f$ be given on $Q_{B}$. Then $f$ is not uniquely determined by these data, even not when restricted to those $x$ with

$$
|x| \leqq a:=d \sin B .
$$

This result is proved by simply constructing a function in the null-space $\mathscr{N}_{B}$ for the so-called interior problem,

$$
\mathscr{N}_{B}=\left\{f \in L_{2}(\Omega): \mathscr{P} f(\alpha, \beta)=0 \text { for almost all }(\alpha, \beta) \in Q_{B}\right\} .
$$

If we chose an even $h \in C_{0}^{\infty}[-1,1]$ with $h \neq 0$ and $h(s)=0$ for $|s|<a$, then we see that

$$
g(\alpha, \beta)=h(d \sin \beta)
$$

is in the range of $\mathscr{P}$ and that, applying Cormack's inversion formula, [9], we get

$$
f(x)=-\frac{1}{\pi} \int_{|x|}^{\infty}\left(s^{2}-|x|^{2}\right)^{-1 / 2} h^{\prime}(s) d s .
$$

This function $f$ is not identically equal to zero and hence a nontrivial member of $\mathscr{N}_{B}$. Instability problems are reported by constructing special elements in the range of $\mathscr{R}$, see $[1,8]$.

Now we start computing a singular value decomposition for $\mathscr{P}$ as mapping between $L_{2}(\Omega)$ into $L_{2}\left(Q_{B}, W\right)$. We denote by

and get

$$
\Psi_{m, l}(\alpha, \beta)=u_{m, l}(d \sin \beta, \omega(\alpha+\beta))
$$

$$
\mathscr{P} v_{m, l}(\alpha, \beta)=\frac{2 \sqrt{\pi}}{\sqrt{m+1}} \Psi_{m, l}(\alpha, \beta)
$$

From this we find

$$
\begin{aligned}
\langle\mathscr{P} * & \left.\mathscr{P} v_{m, l}, v_{n, k}\right\rangle_{L_{2}(\Omega)}=\left\langle\mathscr{P} v_{m, l}, \mathscr{P} v_{n, k}\right\rangle_{L_{2}\left(Q_{B}, W\right)} \\
= & \frac{4 \pi}{\sqrt{(m+1)(n+1)}}\left\langle\Psi_{m, l}, \Psi_{n, k}\right\rangle_{L_{2}\left(Q_{B}, W\right)} \\
= & \frac{4}{\pi \sqrt{(m+1)(n+1)}} d \int_{-B}^{B} \cos \beta w(d \sin \beta) U_{m}(d \sin \beta) U_{n}(d \sin \beta) \\
& \cdot e^{i(l-k) \beta} d \beta \int_{0}^{2 \pi} e^{i(l-k) \alpha} d \alpha \\
= & \delta_{l, k} \frac{4 \pi}{\sqrt{(m+1)(n+1)}} \frac{2}{\pi} \int_{-a}^{a} w(s) U_{m}(s) U_{n}(s) d s .
\end{aligned}
$$

That means that the scalar product is zero for $l \neq k$. On the other hand, according to the singular value decomposition given in Lemma 2.1 and the fact that the 
$v_{m, l}$ form a complete system in $L_{2}(\Omega)$ only those $m, n$ show up that have the same parity as $k$ and are not smaller than $|k|$. Hence we consider for $l=k$ the operators

$$
\left(\tilde{A}_{|k|}\right)_{m n}=\frac{2}{\pi} \int_{-a}^{a} w(s) U_{|k|+2 m}(s) U_{|k|+2 n}(s) d s
$$

and express the value of the scalar product by the operator $A_{|k|}=A_{|k|}(a)$ as

$$
\left\langle\mathscr{P} * \mathscr{P} v_{|k|+2 m, k}, v_{|k|+2 n, k}\right\rangle_{L_{2}(\Omega)}=\left(D_{|k|}^{\top} \tilde{A}_{|k|} D_{|k|}\right)_{m n}=:\left(A_{|k|}\right)_{m n},
$$

where the diagonal operator $D_{k}$ is given by

$$
\left(D_{k}\right)_{m n}=\frac{2 \sqrt{\pi}}{\sqrt{k+2 m+1}}, \quad m \geqq 0 .
$$

Using the relation of the Chebyshev-polynomials with trigonometric functions; i.e.,

$$
U_{m}(\cos \vartheta)=\frac{\sin (m+1) \vartheta}{\sin \vartheta},
$$

we get with $b=\arccos a$

$$
\left(\tilde{A}_{k}\right)_{m n}=\frac{4}{\pi} \int_{b}^{\pi / 2} \sin (k+2 m+1) \vartheta \sin (k+2 n+1) \vartheta d \vartheta
$$

resulting in the representation

$$
\widetilde{A}_{k}=I-T+H_{k}
$$

where $I$ is the identity, $T$ is the Toeplitz-operator

$$
(T)_{m n}=s(m-n)
$$

and $H_{k}$ are the Hankel-operators

$$
\left(H_{k}\right)_{m n}=s(k+1+m+n)
$$

where finally

$$
s(l)=\frac{1}{\pi} \frac{\sin (2 l b)}{l} .
$$

In the full data case the operator $\tilde{A}_{k}$ is, because of $b=0$, the identity and we immediately find the eigenvalue-eigenvector-decomposition of $\mathscr{P} * \mathscr{P}$. The operator $T$ is denoted by Slepian [10] as $\rho\left(\infty, \frac{b}{\pi}\right)$.

Lemma 3.2. The operators $\tilde{A}_{k}$ are linear continuous operators from $l_{2}$ into $l_{2}$ with

$$
\left\|\tilde{A}_{k}\right\| \leqq 1
$$


Proof. Let $k$ be fixed. We represent the operator $\tilde{A}_{k}$ as a product of simple operators. Let $G: l_{2} \rightarrow L_{2}\left(I, w^{-1}\right), I=[-1,1]$ be defined as

$$
l_{2} \ni d=\left(d_{m}\right)_{m=0}^{\infty} \stackrel{G}{\longrightarrow}(G d)(s)=\sqrt{\frac{\overline{2}}{\pi}} w(s) \sum_{k=0}^{\infty} d_{k} U_{k}(s) .
$$

Form Parseval's relation it follows that $G$ has norm 1 . The adjoint operator

$$
G^{*}: L_{2}\left(I, w^{-1}\right) \rightarrow l_{2}
$$

is given by

$$
G^{*} f=\left(\left(G^{*} f\right)_{m}\right)_{m=0}^{\infty}=\left(\sqrt{\frac{2}{\pi}} \int_{-1}^{1} U_{m}(s) f(s) d s\right)_{m=0}^{\infty}=G^{-1} f
$$

With the characteristic function $\chi_{a}$ of the interval $[-a, a], 0<a<1$ we define the time-limiting operator $E_{a}: L_{2}\left(I, w^{-1}\right) \rightarrow L_{2}\left(I, w^{-1}\right)$ simply by

$$
\left(E_{a} f\right)(x)=\chi_{a}(x) f(x) .
$$

It is clear that $\left\|E_{a}\right\|=1$ and $E_{a}^{*}=E_{a}$. Hence the product operator

$$
G_{a}=E_{a} G: l_{2} \rightarrow L_{2}\left(I, w^{-1}\right)
$$

is linear, bounded and we have $G_{a}^{*}=G^{*} E_{a}$. The operator

$$
G_{a}^{*} G_{a}=G^{*} E_{a} E_{a} G=G^{*} E_{a} G=G^{-1} E_{a} G
$$

is a linear and continuous mapping from $l_{2}$ into $l_{2}$ with

$$
\left(G_{a}^{*} G_{a}\right)_{m n}= \begin{cases}\frac{2}{\pi}\left(\frac{\sin ((m+n+2) b)}{m+n+2}-\frac{\sin ((m-n) b)}{m-n}\right) & m \neq n \\ 1-\frac{2}{\pi} b+\frac{1}{\pi} \frac{\sin (2(m+1) b)}{m+1} & m=n\end{cases}
$$

where $b=\arccos (a)$. In order to represent the operator $\tilde{A}_{k}$ with the help of $G_{a}^{*} G_{a}$ we finally need the shift operator

defined as

$$
B_{k}: l_{2} \rightarrow l_{2}
$$

$$
\left(B_{k} d\right)_{m}= \begin{cases}d_{\mu} & \text { for } m=2 \mu+k \\ 0 & \text { otherwise }\end{cases}
$$

Because of $\left\|B_{k} d\right\|=\|d\|$ we see $\left\|B_{k}\right\|=1$. Finally we use $\left(B_{k}^{*} d\right)_{n}=d_{k+2 n}$ to get

$$
\tilde{A}_{k}=B_{k}^{*} G^{-1} E_{a} G B_{k},
$$

which means that the norm of $\widetilde{A}_{k}$ is less or equal 1 . 
Theorem 3.3. The symmetric operators $A_{k}, k \geqq 0$ are compact operators between $l_{2}$ and have norm

$$
\left\|A_{k}\right\| \leqq \frac{4 \pi}{k+1}
$$

Proof. The operators $A_{k}$ are compositions of the continuous operators $\tilde{A}_{k}$ with norm 1 , see Lemma 3.2, and the compact diagonal operators $D_{k}$ with norm $2 \pi^{1 / 2}(k+1)^{-1 / 2}$.

As a consequence we see that the $A_{k}$ have a pure point spectrum. For fixed $k$ we denote the eigenvalues of $A_{k}$ by $\lambda_{k, \mu}$ and the eigenvectors by $x_{k, \mu}$

$$
A_{k} x_{k, \mu}=\lambda_{k, \mu} x_{k, \mu}
$$

These eigenvectors form the unitary transform $X_{k}$ and we use this coordinate transform to find another complete orthonormal system on $L_{2}(\Omega)$ by

$$
\begin{aligned}
& \Phi_{k, \mu}=\sum_{m=0}^{\infty}\left(x_{|k|, \mu}\right)_{m} v_{|k|+2 m, k}, \\
& A_{k, \mu}=2 \sqrt{\pi} \sum_{m=0}^{\infty}\left(x_{|k|, \mu}\right)_{m} \Psi_{|k|+2 m, k} \frac{1}{\sqrt{\lambda_{|k|, \mu}(|k|+2 m+1)}} .
\end{aligned}
$$

Theorem 3.4. The operator $\mathscr{P}: L_{2}(\Omega) \rightarrow L_{2}\left(Q_{B}, W\right)$ has the singular value decomposition

$$
\begin{gathered}
\left\{\Phi_{k, \mu}, \Lambda_{k, \mu}, \tau_{k, \mu}\right\} \\
\tau_{k, \mu}=\sqrt{\lambda_{k, \mu}}
\end{gathered}
$$

with $k \in \mathbf{Z}$ and $m \geqq 0$ and $\Phi, \Lambda$ and $\lambda$ given in (3.8), (3.9), (3.10).

Proof. This follows from the construction of the above used functions.

\section{Stability Considerations}

In this section we study the operators $A_{k}$ and $\tilde{A}_{k}$ in order to get more information on the singular values and the singular functions. Clearly from the Theorems 3.3 and 3.4 we have

$$
\tau_{k, \mu} \leqq 2 \sqrt{\frac{\pi}{k+1}}
$$

The question is now how fast the $\tau_{k, \mu}$ decay as function of $\mu$. 
A first result is provided by the nonuniqueness theorem in Lemma 3.1. There we constructed a circular symmetric function in the null space, whose expansion coefficients are determined with the help of $A_{0}$, a fact that we restate in the following form.

Theorem 4.1. The operator $A_{0}$ is not injective.

Next we consider the high angular frequencies; i.e., large $|k|$.

Theorem 4.2. The operators $\tilde{A}_{k}$ converge for $k \rightarrow \infty$ pointwise to the regular operator $I-T$.

Proof. We show that the $H_{k}$ converge pointwise to 0 . Using

$$
\tilde{A}_{k}=I-T+H_{k}
$$

we get

$$
\left\|H_{k}\right\| \leqq\left\|\tilde{A}_{k}\right\|+\|I-T\| \leqq 2
$$

as a consequence of Theorem 3.3 and the result of Slepian [10]. That means that the $\left\|H_{k}\right\|$ are uniformly bounded. Now let $\left\{e_{i}: i \in \mathbf{N}\right\}$ be the set of canonical unit vectors in $l_{2}$. Then

$$
\begin{aligned}
\left\|H_{k} e_{l}\right\|^{2} & =\sum_{j=0}^{\infty}\left|\sum_{i=0}^{\infty}\left(H_{k}\right)_{j l}\left(e_{l}\right)_{i}\right|^{2}=\sum_{j=0}^{\infty}\left|\left(H_{k}\right)_{j l}\right|^{2} \\
& \leqq \frac{1}{\pi^{2}} \sum_{j=0}^{\infty} \frac{1}{(k+l+j)^{2}} \\
& =\frac{1}{\pi^{2}}\left(\sum_{j=0}^{\infty} \frac{1}{j^{2}}-\sum_{j=0}^{l+k-1} \frac{1}{j^{2}}\right) \\
& =\frac{1}{6}-\frac{1}{\pi^{2}} \sum_{j=0}^{k+1-1} \frac{1}{j^{2}}
\end{aligned}
$$

which converges to 0 for $k \rightarrow \infty$. Hence, $H_{k}$ converges pointwise on $M=\operatorname{span}\left\{e_{i}\right.$ : $I \in \mathbf{N}\}$ to $0 . M$ is dense in $l_{2}$, therefore with the Banach-Steinhaus theorem follows the convergence of $H_{k}$ to 0 .

This result shows that the high angular frequencies are uniquely determined by the truncated projections.

Theorem 4.3. Let $f \in \mathscr{N}_{B}$. Then the two statements are equivalent:

i) $f$ has a finite series expansion,

ii) $f \equiv 0$.

Proof. Obviously i) follows from ii). Now assume that $f$ has a finite series expansion; i.e.,

$$
f=\sum_{m=0}^{M} \sum_{\substack{l=-m \\ m+l \text { even }}}^{m} f_{m l} v_{m !}
$$


Table 5.1. Singular values of the finite section (5 $\times 5)$ approximation of $I-T, H_{k}$ and $\tilde{A}_{k}$ for $a=0.6$

\begin{tabular}{llll}
\hline$I-T$ & $|k|$ & $H_{k}$ & $\tilde{A}_{k}$ \\
\hline 0.989244165313 & & & \\
0.798896832552 & 0 & 0.365234381123 & 0.999984877681 \\
0.268315779263 & & 0.156495747749 & 0977888883151 \\
0.021332998715 & 0.33515367982 & 0.332562371221 \\
0.000391405274 & & 0.007220493882 & 0.003158801636 \\
& 10 & 0.000381971377 & 0.000001726279 \\
& & 0.059402003065 & 0.990985805446 \\
& & 0.049746920445 & 0.777553851287 \\
& & 0.000574205487 & 0.305385603857 \\
& & 0.000301763384 & 0.013663237606 \\
& & 0.000001859933 & 0.000522067577 \\
& & 0.007893909505 & 0.988741851803 \\
& & 0.007264783812 & 0.804485806662 \\
& & 0.000001447839 & 0.261682502510 \\
& 0.000000946172 & 0.022273992662 \\
& & 0.000000000132 & 0.000367399988 \\
\hline
\end{tabular}

and that it is not identically equal to zero, then

$$
\mathscr{P} f(\alpha, \beta)=\sum_{l=-M}^{M} e^{i l(\alpha+\beta)} \sum_{k=0}^{(M-\mid l l) / 2} g_{|l|+2 k . l} V_{|l|+2 k}(\beta)
$$

with

$$
g_{m, l}=\sigma_{m, l} f_{m, l} .
$$

Integrating with respect to $\alpha$ we compute the function

$$
\begin{aligned}
q_{l}(\beta) & =\frac{1}{2 \pi} \int_{0}^{2 \pi} \mathscr{P} f(\alpha, \beta) e^{-i l \alpha} d \alpha \\
& =e^{i l \beta} \sum_{k=0}^{(M-|l|) / 2} g_{|l|+2 k, t} V_{|l|+2 k},
\end{aligned}
$$

hence

$$
e^{-i l \beta} w^{-1}(d \sin \beta) q_{l}(\beta)
$$

is a polynomial in $d \sin \beta$ of degree at most $M$, which has at most $M$ zeroes for $\beta \in[-B, B]$ in contrast to the assumption that $f \in \mathscr{N}_{B}$.

The "bad" functions that are constructed in $[1,8]$ contain essentially components belonging to small singular values. But this information is incomplete as far as this is only a small portion of the possible contributions. The above results give much more insight in this incomplete data problem.

\section{Numerical Results}

The theoretical results of the last sections show different behaviour of the operator $A_{k}$ for small and large $k$. In the following we first present some numerical results for the case of finite section approximations of the compact operators 

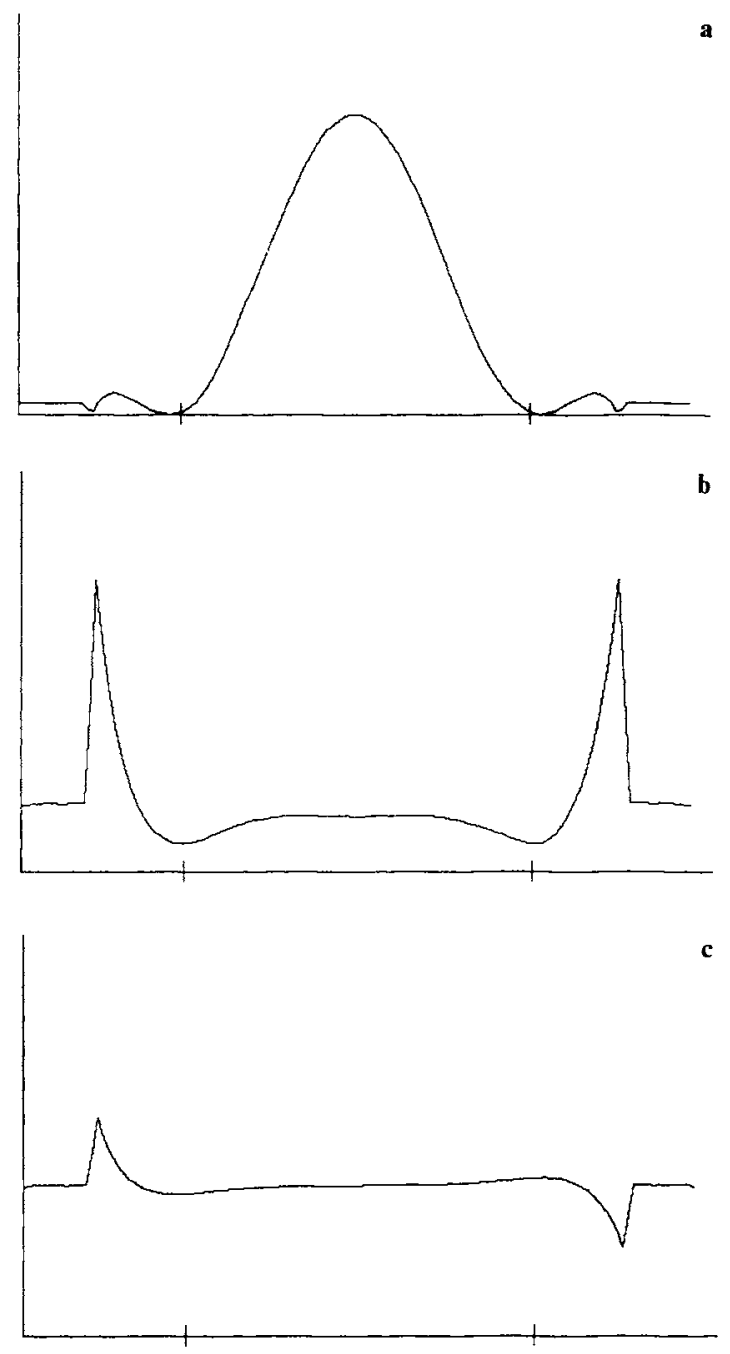

Fig. 5.1 a-c. Numerical approximation to singular functions $\Phi_{5, l}(x, 0)$ for a large and two small singular values. The region of interest is marked

$A_{k}$ respectively for $\tilde{A}_{k}$. In Table 5.1 we compare the singular values of $\tilde{A}_{k}=I-T-H_{k}$ and $H_{k}$ for different values of $k$ and for the regular operator $I-T$. In accordance to Theorem 4.2 we observe that for large $k$ the singular values of $H_{k}$ become smaller and that $\widetilde{A}_{k}$ approaches $I-T$. The result is reported for $M=4$ in the notation of the proof of Theorem 4.3.

Next we consider the singular functions. In Fig. 5.1 we see that for a large singular value the singular function in 5.1 a) is well concentrated within the region of interest. In contrast to that the singular functions belonging to small singular values are flat in that region as predicted by theoretical results in [2, 9], see b), c). But they are large at the border and at the outside of that region. 

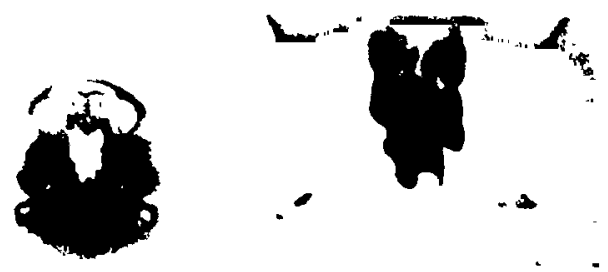

A

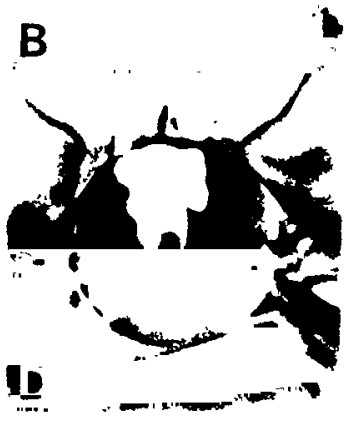

Fig. 5.2a-d. Reconstruction of a head cross section. a full data, b full data zoomed to region of interest, $\mathbf{c}$ reconstruction from the interior 84 rays with standard algorithm, $\mathbf{d}$ reconstruction from interior 84 rays with consistent approximation of the data in the missing range

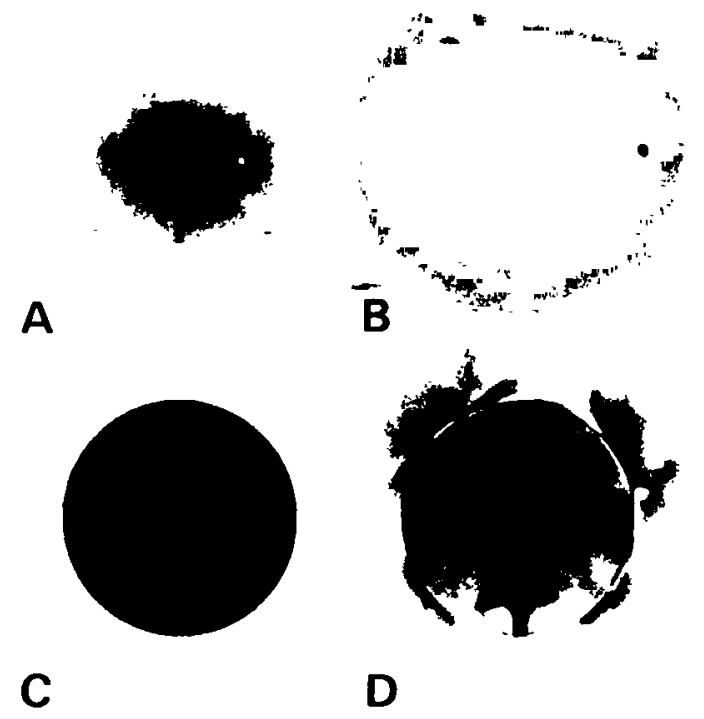

Fig. 5.3a-d. Reconstruction of abdomen cross section. a full data, b full data zoomed to region of interest, $\mathbf{c}$ reconstruction from the interior 164 rays with standard algorithm, $\mathbf{d}$ reconstruction from interior 164 rays with consistent approximation in the missing range 
Of course some of the rays are travelling also through that part, so the data also contain information on that region. Due to that behaviour of the singular values simple algorithms give reasonable reconstructions with a shift in the absolute value of the searched-for densities and they show the contrast of the objects in the interior. Near the border of the region of interest they deteriorate, see $[3,9]$.

We used the truncated singular value decomposition of the above studied operator and computed approximations of the data in the missing range without introducing further information as boundary of the body or so. For realistic tests data from a SIEMENS scanner were provided. In the case of the skull, see Fig. 5.2, we compared the result from the complete set of data with the one where we used from the complete set of 704 rays per direction only the interior 84 rays. In the picture showing the abdomen, see Fig. 5.3 we used the interior 164 rays in order to cover more than the spinal chord. In both cases there are no visible differences of the pictures with complete and truncated data in the region of interest.

\section{References}

1. Bartmann, A.: Unvollstandige Daten in der Computer-Tomographie. Dissertation LMU München 1986

2. Hamaker, C., Smith, K.T., Solmon, D.C., Wagner, S.L.: The divergent beam X-ray transform. Rocky Mountain J. Math. 10, 253-283 (1980)

3. Lewitt, R.M.: Image reconstruction from projections. I. General theoretical considerations. Optik 50, 19-33 (1978)

4. Louis, A.K.: Fast scanning geometries in X-ray computerized tomography. In: Boffi, V., Neunzert, H. (eds.) Applications of mathematics in technology, pp. 324-329. Stuttgart: Teubner 1984

5. Louis, A.K.: T1khonov-Phillips regularızation of the radon transform. In. Hämmerlin, G, Hoffmann, K.H. (eds.) Constructive methods for the practical treatment of integral equations, pp. $211-$ 223. Basel: Birkhäuser 1985

6. Louis, A.K.: Incomplete data problems in X-ray computerized tomography. I- Singular value decomposition of the limited angle transform. Numer. Math. 48, 251-262 (1986)

7. Louis, A.K : Inverse und schlecht gestellte Probleme, 1st Ed. Stuttgart. Teubner 1989

8. Madych, W.R., Nelson, S.A.: Reconstruction from restricted Radon transform data: Resolution and ill-conditionedness. SIAM J Math. Anal. 17, 1447-1453 (1986)

9. Natterer, F.: The mathematics of computerized tomography, 1st Ed New York: Wiley-Teubner 1987

10. Slepian, D.: Prolate spheroidal wave functons. Fourier analysis and uncertainty, V. The Discrete Case. Bell Syst. Techn. J. 57, 1371-1430 (1978) 